DEMOGRAPHIC RESEARCH

VOLUME 31, ARTICLE 28, PAGES 861-888

PUBLISHED 10 OCTOBER 2014

http://www.demographic-research.org/Volumes/Vol31/28/

DOI: 10.4054/DemRes.2014.31.28

Research Article

Migration, sexual networks, and HIV in Agbogbloshie, Ghana

Susan Cassels

Samuel M. Jenness

Adriana A.E. Biney

\section{William Kwabena Ampofo}

\author{
F. Nii-Amoo Dodoo
}




\section{Table of Contents}

1 Introduction $\quad 862$

$2 \quad$ Setting and context 863

$2.1 \quad$ HIV and migration in Ghana 863

$2.2 \quad$ Study setting 865

$3 \quad$ Methods $\quad 866$

3.1 Sampling design 866

$\begin{array}{lll}3.2 & \text { Measurement } & 867\end{array}$

$\begin{array}{lll}3.3 & \text { Statistical analysis } & 869\end{array}$

$4 \quad$ Results $\quad 870$

5 Discussion $\quad 877$

$5.1 \quad$ Future work $\quad 880$

$\begin{array}{lll}5.2 & \text { Limitations } & 880\end{array}$

$\begin{array}{lll}5.3 & \text { Conclusions } & 881\end{array}$

$6 \quad$ Acknowledgements $\quad 881$

$\begin{array}{ll}\text { References } & 882\end{array}$ 


\title{
Migration, sexual networks, and HIV in Agbogbloshie, Ghana
}

\author{
Susan Cassels ${ }^{1}$ \\ Samuel M. Jenness ${ }^{2}$ \\ Adriana A.E. Biney ${ }^{3}$ \\ William Kwabena Ampofo ${ }^{4}$ \\ F. Nii-Amoo Dodoo ${ }^{5}$
}

\begin{abstract}
BACKGROUND

HIV is spread through structured sexual networks, which are influenced by migration patterns, but network-oriented studies of mobility and HIV risk behavior have been limited.
\end{abstract}

\section{OBJECTIVE}

We present a comprehensive description and initial results from our Migration \& HIV in Ghana (MHG) study in Agbogbloshie, an urban slum area within Accra, Ghana.

\section{METHODS}

The MHG study was a population-based cross-sectional study of adults aged 18-49 in Agbogbloshie in 2012. We used a one-year retrospective relationship history calendar to collect egocentric network data on sexual partners as well as migration and shortterm mobility, and tested for prevalent HIV-1/2 infection.

\section{RESULTS}

HIV prevalence was 5.5\%, with prevalence among women (7.2\%) over twice that of men (2.8\%). Three-quarters of residents were born outside the Greater Accra region, but had lived in Agbogbloshie an average of 10.7 years. Only 7\% had moved housing structures within the past year. However, short-term mobility was common. Residents had an average of 7.3 overnight trips in the last year, with women reporting more travel

\footnotetext{
${ }^{1}$ University of California at Santa Barbara, U.S.A. E-Mail: scassels@geog.ucsb.edu.

${ }^{2}$ University of Washington, U.S.A.

${ }^{3}$ University of Ghana.

${ }^{4}$ University of Ghana.

${ }^{5}$ The Pennsylvania State University, U.S.A.
} 
than men. Thirty-seven percent of men and 9\% of women reported more than one sexual partner in the last year.

\section{CONCLUSIONS}

Population-based surveys of migration and sexual risk behavior using relationship history calendars in low-resource settings can produce high quality data. Residents in Agbogbloshie are disproportionately affected by HIV, and have high levels of shortterm mobility. HIV prevention interventions targeted to highly mobile populations in high prevalence settings may have far-reaching and long-term implications.

\section{Introduction}

In Ghana and in many other West African countries, early diffusion of HIV was due in large part to migration and the spatial distribution of sexual networks (Caldwell 1969; Decosas et al. 1995; Oppong 1998; Voeten et al. 2010). In generalized epidemics, migration influences HIV risk in nuanced ways, depending on qualities of the population and HIV epidemic (Cassels et al. 2013; Deane, Parkhurst, and Johnston 2010). Whether HIV prevention strategies should target migrant populations depends on whether migrants are disproportionately affected by HIV or continue to drive ongoing HIV transmission. Therefore innovative methods are needed to study the impact of migration on sexual network structure and HIV diffusion.

HIV is spread through structured sexual networks: socio-demographic patterns of sexual mixing and the timing and sequence of sexual partnerships help determine HIV transmission dynamics (Morris 2004; Morris, Goodreau, and Moody 2007). The characteristics of sexual partnerships depend on migration patterns as well, but network-oriented studies of mobility and HIV risk behavior have been limited. Through both geographical moves in space and changes in sexual networks, migration and mobility can influence partnership formation and dissolution, the characteristics of partnerships, and behavior within partnerships.

Countries with generalized epidemics like Ghana afford an opportunity to investigate the role that migration plays in HIV transmission. Ghana's national HIV prevalence is near 2\% (Ghana AIDS Commission 2012), but HIV prevalence varies significantly by sex, socioeconomic status, and region. Young women in the national capitol city Accra suffer disproportionately from HIV, with a prevalence measured near 8.3\% (Duda et al. 2005). Migration is common in Ghana; more than $50 \%$ of the population were either born outside of their current place of residence or have lived outside for a year or more (Ghana Statistical Service (GSS) 2009). Much of the past work on migration and HIV has taken place in high-HIV-prevalence countries. With the 
bulk of what we know about HIV-related behavior coming from research in highprevalence contexts, such inquiry into the role of migration and HIV transmission dynamics in Ghana will balance our understanding of behavior and help validate existing knowledge.

We present a comprehensive description and initial results from our recently completed Migration \& HIV in Ghana (MHG) study of migration, sexual networks, and HIV risk behavior in Agbogbloshie, an urban slum area within Accra, Ghana. The MHG study was an innovative population-based cross-sectional study of adults aged 18-49 in Agbogbloshie in 2012. We used a one-year retrospective relationship history calendar (RHC) to collect egocentric data on sexual partners as well as on migration and short-term mobility (Luke, Clark, and Zulu 2011). We supplemented the RHC with structured questions on migration, mobility, socio-demographics, and sexual history. Lastly, we collected dried blood spots, which we tested for prevalent HIV-1/2 infection. In this paper we first describe the history of HIV and migration in Ghana, and then specifically describe our study setting. Second, we describe our sample design, measures, and statistical analysis. After that we present initial results from the study. We end with a discussion of lessons learned from field implementation, and suggestions for future work.

\section{Setting and context}

\subsection{HIV and migration in Ghana}

After the first case of HIV was reported in Ghana in 1986 (Ishikawa et al. 1988; Masuda et al. 1988) the number of cases continued to increase until 2003, with prevalence peaking near 3.6\%. National adult HIV prevalence currently is declining: in 2011 it was near 2\% (Ghana AIDS Commission 2012). The first reports of HIV in Ghana were of HIV-2, but competition with HIV-1 was already occurring during the 1990s (AduSarkodie, Tetteh, and AppiahDenkyira 1996). The proportion of AIDS due to a single infection with HIV-2 decreased, and by the late 1990s HIV-1 comprised

most HIV infections in Ghana (Brandful et al. 1997; Brandful et al. 1998). As reported by the 2003 Ghana Demographic and Health Survey (DHS), 95\% of HIV cases were HIV-1 only, $4.5 \%$ of cases were dually infected with HIV-1 and HIV-2, and only $0.5 \%$ of cases were HIV-2 only (Akwara et al. 2005). The shift to an HIV-1 epidemic in Ghana may be due to HIV-1 outcompeting HIV-2 in population transmission dynamics (i.e., natural selection) (de Silva, Leligdowicz and Rowland-Jones 2012; de Silva et al. 2013), but also mobility linking Ghanaians to areas endemic with HIV-1 (Tatem et al. 2012). 
In the early 2000s most HIV infections in Accra originated from contact with sex workers (Cote et al. 2004), with more recent spread from these core risk groups to the general population. The most recent modes-of-transmission model suggests that the epidemic is characterized as heterosexually driven, while paid sex is estimated to be the cause of 32\% of all new infections (UNAIDS 2010). However, HIV prevalence in Ghana still varies by region, sex, and age, with highest prevalence found among women, persons in their early thirties, and in the Central and Eastern administrative regions (Ghana AIDS Commission 2012).

Ghana's early HIV epidemic developed uniquely and has been linked to migration patterns (Agyei-Mensah 2001; Oppong 1998). Ghana was an attractive destination for in-migrants in the years after independence in 1957 (Nyame, Grant, and Yakovleva 2009; Stock 2013). During the economic crisis in the early 1980s, a few years before the first HIV cases were reported in Ghana, many more Ghanaians were out-migrating, often to Europe, North America, or Western African countries with stable economies at the time such as Nigeria and Cote d'Ivoire. However, more than a million Ghanaian migrants in Nigeria were forced to return to Ghana when Nigeria experienced an economic downturn in 1983 (Owusu 1995).

Many studies suggest that Ghanaian female commercial sex workers migrating to and from Cote d'Ivoire contributed to the early HIV epidemic (Anarfi 1993; Decosas 1995; Decosas et al. 1995; Djomand et al. 1995; Oppong 1998). Most HIV cases had been found in people with a history of residence or extended visit outside Ghana (Oppong 1998). A large study of migration, sexual risk behavior, and HIV conducted in 1991 hypothesized that migration removes social restrictions to having multiple sex partners and suggested that circular migration played an important role in connecting sexual networks in Ghana (Anarfi 1993). A comprehensive paper on the geographical patterns of HIV in Ghana describes the role of migration in the development of the epidemic (Agyei-Mensah 2001).

Patterns of internal migration in Ghana are changing and current migration characteristics may affect HIV transmission dynamics differently. Typically, male migrants from the Northern region served as laborers in agriculture, mining, and industrial sectors along the southern coast (Caldwell 1969; Oberhauser and Yeboah 2011). Now, migration destinations are diversifying, labor flows are transforming, and women are moving independently of their families due to increasing social and economic independence as well as worsening female-dominated livelihoods in the Northern region. Research on female head-porters (known in Ghana as Kayayei) highlights common socio-spatial patterns of contemporary female migration in Ghana, from the rural agricultural-based north to the urban south (Awumbila and ArdayfioSchandorf 2008; Oberhauser and Yeboah 2011). Lastly, rates of urbanization might be slowing for many cities in sub-Saharan Africa (Potts 2012) while circular migration is 
increasing, which has reduced the contribution of in-migration to urban growth. These trends are largely the result of declining economic opportunities in many urban areas, reflecting crises in urban poverty and livelihood insecurity (Potts 2009, 2012). Therefore contemporary studies on migration and HIV must consider the context and characteristics of circular migration.

A number of recent articles in the migration and HIV literature highlight the need to investigate different types of mobility and account for sexual network characteristics and epidemiological context (Deane et al. 2010). These demonstrate useful methods to capture the complexities in migration and HIV research, such as aligning timing and exposure (Anglewicz 2012) and in-depth understanding of context (Camlin et al. 2014). Our current work is poised to contribute to the network-dyadic conceptual framework for migration and HIV studies (Cassels, Jenness, and Khanna 2013 ). The networkdyadic conceptual model for migration and HIV suggests that migration can influence individual-level characteristics (i.e., traits of a migrant compared to others in the community), can affect partnership formation and dissolution, and can influence the sexual network itself by expanding the size of the network or bridging two otherwise distinct networks (Cassels et al. 2013 ).

Despite high rates of urbanization and circular migration in Ghana, current literature on migration and health, especially infectious disease epidemiology, is sparse. Research has linked rates of lower fertility to urbanization (White et al. 2008) and neighborhood influences (Weeks et al. 2010), but these studies do not explicitly measure sexual behavior. The studies on the female migrant head porters mentioned above (Awumbila and Ardayfio-Schandorf 2008; Oberhauser and Yeboah 2011) thinly address the important issue of sexual health for these young girls and women. An analysis of the 2008 Ghana DHS examined predictors of unprotected sex but did not examine mobility, and did not find meaningful associations with place of residence (rural/urban) or region either (Kumi-Kyereme, Tuoyire, and Darteh 2013). Our study builds upon recent conceptual and methodological advances and updates knowledge on migration and HIV in Ghana.

\subsection{Study setting}

Our study setting, Old Fadama (generally referred to as Agbogbloshie), is a suburb of Accra made up of five Enumeration Areas (EAs, similar to census tracks in the United States) defined by the Ghana Statistical Service in 2010 as: 'Efo Market' (0304303143), 'Presbyterian Church' (0304303139), 'Happy Corner Spot' (0304303140), 'H/No. Blk 12' (0304303142), and ' $31^{\text {st }}$ December Market' (0304303138). There are 1,731 EAs in the Accra metropolitan area (AMA), which is the largest district within the Greater 
Accra region, the most populous of the 10 regions comprising Ghana. Researchers from the U.S. and Ghana have created digital boundary files of EAs in the AMA; our study site is in the Korley Dudor locality: http://www.geointerest.frih.org/accra.swf (Verutes et al. 2012; Weeks et al. 2010; Weeks et al. 2012; Weeks et al. 2007).

Agbogbloshie is a densely settled, resource-poor setting; most residents lack access to clean water and sanitation. According to the 2010 census, the population size of Agbogbloshie is 8,305 (54\% female and 46\% male), of whom 5,466 are aged 15-49 (same sex distribution) (Ghana Statistical Service 2012). Unlike such neighboring areas as Ussher Town and James Town, which are predominantly inhabited by residents of the Ga ethnicity, Agbobloshie residents come from many regions in Ghana and represent a wide range of ethnicities. Residential dwellings are a mix of concrete structures and wooden shacks; often many unrelated families live within one structure. Agbogbloshie is situated near the banks of the Korle Lagoon, northwest of the Accra Central business district, and is prone to flooding, which has been responsible for significant morbidity and mortality (Joint UNEP/OCHA Environment Unit 2011).

Finally, Agbogbloshie is home to Ghana's largest commercial fresh produce market, which attracts traders from all over the country, as well as seasonal migrants who engage in trade or transport goods for clients in the congested market area (Oberhauser and Yeboah 2011). Agbogbloshie has recently garnered international attention for its large electronics waste processing site, and the resulting burning of plastics is an ongoing environmental hazard to Agbogbloshie residents (Agyei-Mensah and Oteng-Ababio 2012; Arguello et al. 2013; Hugo 2010; Oteng-Ababio 2010, 2012). Recently, the market has suffered from fires from unknown causes, which have caused severe damage and affected livelihoods.

\section{Methods}

\subsection{Sampling design}

Our Migration \& HIV in Ghana (MHG) study was a cross-sectional study of sexually active adults within Agbogbloshie, Ghana, during 2012. We used a three-stage cluster randomized sampling scheme to enumerate and sample subjects into the study with the goal of population-based inference on key demographic and epidemiologic outcomes. An earlier study in 2010 within Ga Mashie and Agbogbloshie, the Urban Health and Poverty in Accra project (previously referred to as Edulink), systematically censused the neighborhood to map and enumerate all housing structures, unique households (distinct familial units) within each structure, and the number of residents in each household. Housing structures were physically marked and the name of each household 
head was recorded on the census listing (see (Dodoo 2012) for further details on listing procedures).

We used this census to sample primary 'index' subjects. First households, then adults within households were randomly selected. With a target sample size $(n=475)$ for index subjects, a total number of households in the census $(n=1158)$, and an expected study completion rate (70\%), we randomly sampled $60 \%$ of households, then randomly sampled one member per household. Given differences in household size, we used a weighting scheme to address differential inclusion probabilities across households: the probability for selecting any household was proportional to the size of that household.

In households in which the primary index subject was married or had a cohabiting partner, the subject was asked to refer those partners into the study. Recruitment tracking forms linked the partner study data to the index data. For both index and partner recruitment, study staff attempted at least three visits to the sampled household, and recorded possible reasons for non-response for the household, including structure demolition and long-term travel of the selected household member.

The eligibility criteria were current residence in the selected Agbogbloshie household, age 18 to 49 years old, and lifetime history of consensual sexual intercourse. Referred martial partners who were not cohabiting with the index subject were not required to live within Agbogbloshie, but all did in the end. The Institutional Review Boards of the University of Washington and the Noguchi Memorial Institute for Medical Research, University of Ghana, approved the study protocols.

\subsection{Measurement}

Study procedures included a standardized survey administered by trained field staff, and a diagnostic HIV-1/2 test. The survey was pre-tested in 2011 on a small sample $(n=39)$ of the same target population to refine question wording. The survey was divided into three parts: 1) structured questions for basic demographics, summary travel and sexual behavior, and other information; 2) an event-history calendar for detailed travel and sexual history over the previous year; and 3) semi-qualitative questions to obtain more detailed travel history in a different format.

Demographic questions included marital and socioeconomic status. Medical history questions included prior knowledge of diagnosis with HIV and other sexually transmitted infections. A series of structured questions captured lifetime and recent migration and travel patterns, including the place of birth, length of time living in Agbogbloshie, number of residences within Accra, and summary measures of number of trips and reasons for that travel. Trips were defined as any overnight travel away 
from home, even within Agbogbloshie. Structured partnership questions queried the lifetime history of sexual activity (age of debut and number of lifetime partners) and number of past-year partners. Partners were defined as persons with whom the subject had engaged in consensual vaginal or anal intercourse. Detailed questions on up to the past three partners within the prior year, including relative age and socioeconomic status, were then asked.

For the event history calendar, questions and responses were addressed interactively with the subject, with the events over the year entered into the survey tool in order of recall related to key milestones (e.g., childbirth and holidays). Responses were obtained for each of the 12 months prior to the survey to obtain retrospective longitudinal data. Sexual data here were limited to the last three partners, including the duration and type of the partnership, the frequency of protected and unprotected sexual activity within the partnership, and geographic location of the activity within Ghana. Arrows at the start and end month were used to indicate left-censoring (started before the earliest month) and right-censoring (ongoing past the day of the interview), respectively.

To illustrate our calendar tool, Figure 1 shows the calendar format with artificial example data for three of the survey questions: partnership duration over the year, type of relationship, and frequency of sexual activity within the month. Data were obtained for each month, for each of the past three partners in the past year (although most study participants only reported one past-year partner). In this example, the duration of the relationship was 12 months, ongoing. The type of relationship question is recorded in numbers corresponding to categories stated elsewhere in the survey, with higher numbers indicating more causal partnerships ( 4 = dating) and lower numbers indicating steadier partnerships $(1=$ married $)$. For the frequency of sex question, we asked how many distinct acts or rounds of sex the subject had had with the partner within that month.

Figure 1: Event history calendar scheme

\begin{tabular}{|c|c|c|c|c|c|c|c|c|c|c|c|c|c|}
\hline & & \multicolumn{5}{|c|}{2011} & \multicolumn{7}{|c|}{2012} \\
\hline & & Aug & Sep & Oct & Nov & Dec & Jan & Feb & Mar & Apr & May & Jun & Jul \\
\hline D12 & Duration & & & & & & & & & & & & $\longrightarrow$ \\
\hline D16 & Type of Rel. & 4 & 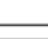 & -1 & 3 & _ & _ & 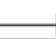 & 2 & 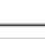 & -1 & 1 & $\longrightarrow$ \\
\hline D22 & Freq. Sex & 8 & 8 & 8 & 8 & 0 & 0 & 8 & 4 & 2 & 0 & 0 & 0 \\
\hline
\end{tabular}

All subjects who completed the survey were asked to voluntarily take a diagnostic HIV test, although survey participants could decline testing. Dried blood spots were 
collected in the field on standard filter paper and maintained in refrigerators before delivery to the Department of Virology, Noguchi Memorial Institute for Medical Research, University of Ghana, Legon, for processing. Serum was tested with the INNO-LIA $^{\mathrm{TM}}$ HIV 1/2 test (Innogenetics, Belgium). The test platform has been shown to have good sensitivity and specificity for diagnosis and HIV type differentiation. All subjects who tested for HIV were asked to return in one week to receive their test results, which were provided by a trained nurse counselor in accordance with local standards. Subjects testing HIV positive were referred to appropriate medical care. Results from subjects with an indeterminate test were not analyzed here. All subjects who took the survey received an incentive of 15 Ghana Cedis, and those who HIVtested received an additional 10 Ghana Cedis as token compensation for time and transport.

\subsection{Statistical analysis}

The MHG data required a sophisticated statistical analysis approach, due to the complex study design, longitudinal measurement methods, and dependent network outcomes. Firstly, statistical methods for the survey design were used to account for the unequal selection probabilities of the target population members, as well as inflation of variance associated with cluster sampling. Field staff collected the household size for all age-eligible household members that were sampled and successfully contacted (with one adult from each household asked to enumerate). A quasi-Poisson regression model with flexible variance parameterization was then used to estimate the relationship between the household size of all members, as collected in the 2010 neighborhood census, to this newly collected household size. This model fit the data well $(p<0.001)$. Predictions from the model were used both to understand the general relationship between the old and new household size, and also to impute new household size for 27 households with missing data (staff did not collect this data on the first three days of the study). For outcomes, we use a generalization of the standard Horvitz-Thompson estimator (here, for a continuous mean) (Lumley 2004):

$$
\hat{\mu}=\frac{1}{N} \sum_{i=1}^{v} y_{i} \frac{\omega_{i}^{n}}{\omega_{i}^{o}}
$$

where $y_{i}$ is the outcome of interest, $N$ is the total population size, $v$ is the total sample size of index subjects, $\omega_{i}$ are the weights (the inverse of the selection probability), with the new weight, $\omega^{n}$, in the numerator and the old weight, $\omega^{o}$, in the denominator. Although this is a weighted simple random sample, since only one subject is selected 
from each selected household, variance may be underestimated without adjustment for the dependency of households within the same physical structure. We therefore use robust cluster-based standard errors for variance estimation (Lumley 2004).

From this study, three types of analyses will be conducted (the first of which is featured here). First, descriptive estimates of key demographic and epidemiologic outcomes are presented using statistical summaries of means, proportions, and standard deviations around the means. Analytic statistics will be used to compare outcomes across groups, such as the prevalence of migration by sex, with $95 \%$ confidence intervals used to express the bounds around the estimates. When comparing outcomes by sex, we generally avoid null-hypothesis significance testing because of the potential inflation of type-I error across all the outcomes, as well as the primarily descriptive focus of this paper. However, we use weighted linear and logistic regression to compare cumulative partner number and HIV-1 infection by sex, respectively. Second, in future work we will use regression methods for the analysis of longitudinal data to investigate whether there are cyclical time trends for the outcomes, and whether any such trends may be predicted by demographic and behavioral variables. Lastly, empirical analyses from this study will be used to parameterize mathematical models for HIV transmission dynamics in sub-Saharan Africa. For this, the estimates derived from the first two types of analysis will be fed into dynamic models that explicitly simulate the network structure of the population as HIV spreads through it (e.g., Goodreau et al. 2012). We describe these latter two approaches further in the discussion.

\section{Results}

Overall, we recruited 590 subjects, of whom 106 were referred partners in linked cohabiting partnerships. From the 694 households selected for first-stage recruitment we recruited 484 index subjects, which represented a completion rate of $70 \%$. The reasons for non-recruitment were ineligibility of all household members due to age or lack of sexual activity (6.6\%), refusal of participation (1.9\%), long-term travel (3.9\%), lack of success locating the subject (14.1\%), and other reasons including the demolition of the housing structure (4.2\%). The response rate for HIV testing (survey participants could decline testing) was $91.0 \%$. These results will present data only on the index subjects.

In a Poisson model predicting the new (age-eligible, sexually active) household size from the old census (all members regardless of age or sexual history), there was a positive, statistically significant relationship for the coefficient $(\beta=0.058 ; p<0.001)$. More clearly, the predicted values for households with old sizes of 1,5 , and 10 members were 1.6, 2.0, and 2.7, respectively. This resonates with our qualitative 
assessment, as many larger households tended to have two adult parents with several cohabiting children and elderly family members. Using the old size for the sample weighting would result in overrepresentation of these large households, which could bias the outcomes.

Table 1 shows descriptive demographic and epidemiologic characteristics of our target population. Over half of the population was female (58.4\%) and below the age of 30 (54.4\%). One-quarter reported being married (29\%), and polygamous marriage was uncommon (0.8\%). Qualitatively, marriage was often ambiguously defined: many of those who reported being married had not had a legal ceremony; thus some reporting marriage may legally be cohabiting. We observed a wide span of education levels, with nearly one in seven reporting secondary education or higher. Similar to Ghana overall, Agbogbloshie residents were heavily Christian, with the Pentecostal sect the most common (46.6\%). Finally, 11.6\% reported a non-HIV sexually transmitted infection diagnosis in their lifetime, and $1.9 \%$ in the prior year. Gonorrhea was the dominant STI among those reporting an infection. Overall, 5.5\% of those who tested were infected with HIV-1, and prevalence was higher among women (7.2\%) than men (2.8\%), with moderate statistical evidence for a difference in the population $(p=0.09)$. We observed no HIV-2 infection in our sample.

In Figure 2 and Table 2 we highlight key outcomes related to migration and mobility. Subjects were asked the city or town of their birth, which we then geocoded as latitude/longitude coordinates. For $8 \%$ of subjects we were unable to geo-locate their hometown (mostly due to very small localities); in that case we used the centroid of their region of birth (which was never missing). Ghana is divided into 10 administrative regions, with Agbogbloshie located in the Greater Accra region. Greater Accra was the single most common region of birth (25.0\%), but this implies three-quarters were born outside the region. Overall, $1.1 \%$ of the population was born in a foreign country. For each subject we calculated the distance along major road networks between the birthplace and Agbogbloshie (Kahle and Wickham 2013). The mean distance was 230 kilometers, with a standard deviation of $247 \mathrm{~km}$. The median distance was $165 \mathrm{~km}$, and 5\% were born over $754 \mathrm{~km}$ from Agbogbloshie. In Figure 2 we map the birthplace of those born within Ghana, with point color representing the subject's sex (red circles = women; blue circles $=$ men). The points were randomly jittered to reduce overplotting. 
Cassels et al.: Migration, sexual networks, and HIV in Agbogbloshie, Ghana

Table 1: Demographic and epidemiologic characteristics of sexually active adults in Agbogbloshie, Accra, Ghana, 2012

\begin{tabular}{|c|c|c|c|c|c|c|}
\hline & \multicolumn{2}{|r|}{ Total } & \multicolumn{2}{|r|}{ Males } & \multicolumn{2}{|r|}{ Females } \\
\hline & $\%$ & $95 \% \mathrm{Cl}$ & $\%$ & $95 \% \mathrm{Cl}$ & $\%$ & $95 \% \mathrm{Cl}$ \\
\hline \multicolumn{7}{|l|}{ Gender } \\
\hline Male & 41.6 & $35.7-47.5$ & - & - & - & - \\
\hline Female & 58.4 & $52.5-64.2$ & - & - & - & - \\
\hline \multicolumn{7}{|l|}{ Age } \\
\hline $18-29$ & 54.4 & $48.8-60.1$ & 52.9 & $44.8-61.0$ & 55.5 & $48.1-62.9$ \\
\hline $30-39$ & 28.8 & $23.8-33.8$ & 31.5 & $24.1-39.0$ & 26.8 & $20.5-33.1$ \\
\hline $40-49$ & 16.8 & $11.9-21.7$ & 15.6 & $9.4-21.7$ & 17.7 & $11.3-24.1$ \\
\hline \multicolumn{7}{|l|}{ Marital Status } \\
\hline Never Married & 42.5 & $36.7-48.3$ & 43.6 & $34.5-52.7$ & 41.7 & $34.4-49.1$ \\
\hline Cohabiting & 18.8 & $13.6-24.0$ & 17.9 & $10.9-24.9$ & 19.4 & $12.4-26.4$ \\
\hline Married Mono. & 28.2 & $23.1-33.3$ & 30.9 & $23.1-38.7$ & 26.3 & $20.2-32.4$ \\
\hline Married Poly. & 0.8 & $0.1-1.6$ & 0.7 & $0.0-1.5$ & 0.9 & $0.0-2.0$ \\
\hline Div./Sep./Widow. & 9.6 & $6.4-12.9$ & 6.9 & $3.4-10.3$ & 11.6 & $6.9-16.4$ \\
\hline \multicolumn{7}{|l|}{ Education } \\
\hline None & 13.7 & $10.0-17.4$ & 8.4 & $4.1-12.7$ & 17.5 & $12.5-22.6$ \\
\hline Primary & 21.8 & $16.8-26.8$ & 18.4 & $12.3-24.3$ & 24.3 & $17.6-30.9$ \\
\hline Middle & 49.8 & $44.5-55.0$ & 50.2 & $42.6-57.7$ & 49.5 & $41.8-57.1$ \\
\hline Secondary & 12.8 & $9.1-16.5$ & 19.1 & $12.1-26.0$ & 8.3 & $4.7-11.9$ \\
\hline Higher & 1.9 & $0.03-3.6$ & 0.4 & $0.0-1.0$ & 4.0 & $0.2-7.8$ \\
\hline \multicolumn{7}{|l|}{ Religion } \\
\hline Catholic & 9.3 & $5.2-13.3$ & 13.0 & $8.2-17.8$ & 6.6 & $1.4-11.8$ \\
\hline Presbyterian & 8.8 & $5.9-11.7$ & 6.3 & $2.8-9.7$ & 10.6 & $6.0-15.1$ \\
\hline Pentecostal & 46.6 & $40.9-52.3$ & 35.8 & $27.8-43.8$ & 54.3 & $46.8-61.8$ \\
\hline Muslim & 13.7 & $9.3-18.2$ & 14.1 & $7.8-20.5$ & 13.4 & $7.6-19.2$ \\
\hline Other & 12.6 & $9.2-16.0$ & 13.5 & $7.9-19.1$ & 11.9 & $7.5-16.2$ \\
\hline None & 9.1 & $5.8-12.3$ & 17.2 & $11.3-23.1$ & 3.2 & $0.5-5.8$ \\
\hline \multicolumn{7}{|l|}{ HIV-1 Infection } \\
\hline Infected & 5.5 & $2.8-8.1$ & 2.8 & $0.2-5.4$ & 7.2 & $3.1-11.3$ \\
\hline Uninfected & 94.5 & $91.9-97.2$ & 97.2 & $94.6-99.8$ & 92.8 & $88.7-96.9$ \\
\hline \multicolumn{7}{|l|}{ Other STI Dx } \\
\hline Ever & 11.6 & $7.1-16.1$ & 22.9 & $15.0-30.8$ & 3.5 & $0.1-6.9$ \\
\hline Past Year & 1.9 & $0.3-3.6$ & 2.8 & $0.3-5.3$ & 1.3 & $0.0-3.5$ \\
\hline
\end{tabular}


Figure 2: $\quad$ Map of birthplace by sex among study subjects, Ghana (upper panel) and Greater Accra (lower panel)
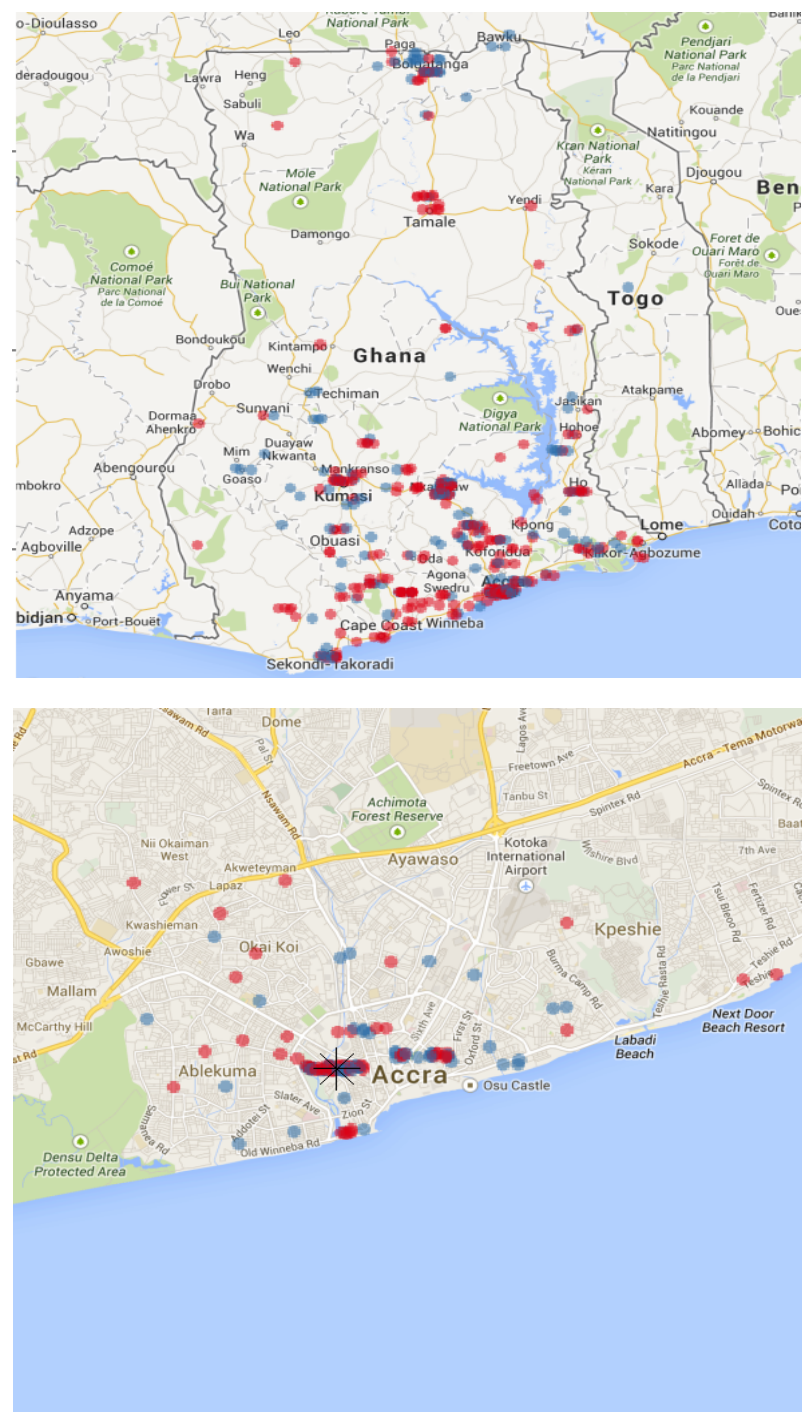

Note: Red dots signify women and blue dots men. The lower panel includes a star indicating the location of Agbogbloshie in Accra. 
Cassels et al.: Migration, sexual networks, and HIV in Agbogbloshie, Ghana

Table 2: $\quad$ Migration, mobility, and sexual history among sexually active adults in Agbogbloshie, Accra, Ghana, 2012

\begin{tabular}{|c|c|c|c|c|c|c|}
\hline & \multicolumn{2}{|r|}{ Total } & \multicolumn{2}{|r|}{ Men } & \multicolumn{2}{|r|}{ Women } \\
\hline & $\%$ / mean & $95 \% \mathrm{Cl}$ & $\% /$ mean & $95 \% \mathrm{Cl}$ & $\%$ / mean & $95 \% \mathrm{Cl}$ \\
\hline \multicolumn{7}{|l|}{ Migration } \\
\hline \multicolumn{7}{|l|}{ Region of Birth } \\
\hline Western & $5.0 \%$ & $2.5 \%-7.5 \%$ & $5.0 \%$ & $1.7 \%-8.3 \%$ & $5.0 \%$ & $1.4 \%-8.7 \%$ \\
\hline Central & $12.3 \%$ & $7.9 \%-16.7 \%$ & $7.2 \%$ & $2.4 \%-11.9 \%$ & $15.9 \%$ & $9.9 \%-21.9 \%$ \\
\hline Greater Accra & $24.5 \%$ & $19.5 \%-29.5 \%$ & $31.9 \%$ & $23.3 \%-40.4 \%$ & $19.3 \%$ & $13.9 \%-24.6 \%$ \\
\hline Volta & $9.1 \%$ & $5.6 \%-12.7 \%$ & $11.1 \%$ & $5.9 \%-16.2 \%$ & $7.8 \%$ & $4.1 \%-11.5 \%$ \\
\hline Eastern & $17.6 \%$ & $13.4 \%-21.7 \%$ & $15.3 \%$ & $9.7 \%-21.0 \%$ & $19.1 \%$ & $13.3 \%-24.9 \%$ \\
\hline Ashanti & $13.5 \%$ & $9.4 \%-17.6 \%$ & $11.9 \%$ & $6.8 \%-16.9 \%$ & $14.7 \%$ & $8.7 \%-20.6 \%$ \\
\hline Brong Ahafo & $2.4 \%$ & $0.8 \%-3.9 \%$ & $3.0 \%$ & $0.5 \%-5.4 \%$ & $2.0 \%$ & $0.1 \%-3.8 \%$ \\
\hline Northern & $3.7 \%$ & $1.3 \%-6.0 \%$ & $1.2 \%$ & $0.0 \%-2.5 \%$ & $5.5 \%$ & $1.6 \%-9.4 \%$ \\
\hline Upper East & $9.8 \%$ & $5.0 \%-14.5 \%$ & $12.1 \%$ & $6.2 \%-18.0 \%$ & $8.1 \%$ & $2.5 \%-13.7 \%$ \\
\hline Upper West & $1.1 \%$ & $0.0 \%-2.9 \%$ & $0.0 \%$ & - & $2.0 \%$ & $0.0 \%-4.9 \%$ \\
\hline Foreign Country & $1.1 \%$ & $0.2 \%-2.0 \%$ & $1.5 \%$ & $0.0 \%-3.3 \%$ & $0.7 \%$ & $0.0 \%-1.6 \%$ \\
\hline $\begin{array}{l}\text { Birthplace KM from } \\
\text { Agbogbloshie }\end{array}$ & 230.3 & $195.8-264.8$ & 218.6 & $175.7-261.5$ & 238.7 & $194.0-283.4$ \\
\hline Years in Agbogbloshie & 10.7 & $9.4-12.0$ & 12.8 & $10.8-14.8$ & 9.3 & $8.0-10.6$ \\
\hline Years in Structure & 7.6 & $6.5-8.8$ & 8.5 & $6.6-10.5$ & 7.0 & $5.8-8.2$ \\
\hline $\begin{array}{l}>1 \text { Agbogbloshie } \\
\text { Structure }^{1}\end{array}$ & $6.6 \%$ & $3.2 \%-10.0 \%$ & $5.0 \%$ & $1.6 \%-8.4 \%$ & $7.8 \%$ & $2.3 \%-13.2 \%$ \\
\hline \multicolumn{7}{|l|}{ Mobility } \\
\hline Number of Trips ${ }^{1}$ & 7.3 & $4.0-10.6$ & 6.1 & $2.0-10.2$ & 8.1 & $3.4-12.9$ \\
\hline Travel $>1$ month $^{1,2}$ & $16.0 \%$ & $11.1 \%-20.9 \%$ & $13.1 \%$ & $6.7 \%-19.4 \%$ & $18.0 \%$ & $10.6 \%-25.5 \%$ \\
\hline Travel Regularly $^{1,2}$ & $26.8 \%$ & $21.0 \%-32.6 \%$ & $25.3 \%$ & $17.1 \%-33.5 \%$ & $27.8 \%$ & $19.4 \%-36.2 \%$ \\
\hline \multicolumn{7}{|l|}{ Sexual History } \\
\hline No. Lifetime Partners & 4.4 & $3.6-5.2$ & 6.4 & $4.6-8.2$ & 2.9 & $2.7-3.2$ \\
\hline Age of Sexual Debut & 18.2 & $17.9-18.6$ & 18.7 & $18.0-19.3$ & 17.9 & $17.5-18.4$ \\
\hline \multicolumn{7}{|l|}{ No. Partners ${ }^{1}$} \\
\hline 0 & $12.6 \%$ & $8.5 \%-16.7 \%$ & $10.6 \%$ & $5.6 \%-15.7 \%$ & $14.0 \%$ & $8.2 \%-19.8 \%$ \\
\hline 1 & $66.7 \%$ & $61.3 \%-72.2 \%$ & $52.2 \%$ & $43.9 \%-60.5 \%$ & $77.1 \%$ & $70.1 \%-84.0 \%$ \\
\hline 2 & $12.9 \%$ & $9.2 \%-16.6 \%$ & $23.6 \%$ & $16.3 \%-30.8 \%$ & $5.3 \%$ & $2.6 \%-8.0 \%$ \\
\hline $3+$ & $7.8 \%$ & $4.8 \%-10.8 \%$ & $13.6 \%$ & $7.9 \%-19.2 \%$ & $3.6 \%$ & $1.0 \%-6.2 \%$ \\
\hline \multicolumn{7}{|l|}{ No. Unprot. Partners ${ }^{1}$} \\
\hline 0 & $18.7 \%$ & $13.9 \%-23.5 \%$ & $17.4 \%$ & $10.9 \%-23.8 \%$ & $19.7 \%$ & $13.1 \%-26.3 \%$ \\
\hline 1 & $66.4 \%$ & $60.8 \%-72.1 \%$ & $58.1 \%$ & $49.7 \%-66.6 \%$ & $72.3 \%$ & $65.0 \%-79.6 \%$ \\
\hline 2 & $9.8 \%$ & $6.6 \%-13.0 \%$ & $15.6 \%$ & $9.7 \%-21.4 \%$ & $5.7 \%$ & $2.6 \%-8.8 \%$ \\
\hline $3+$ & $5.1 \%$ & $2.9 \%-7.2 \%$ & $8.9 \%$ & $4.5 \%-13.4 \%$ & $2.3 \%$ & $0.3 \%-4.3 \%$ \\
\hline
\end{tabular}

Note: ${ }^{1}$ In the past 12 months

${ }^{2}$ Among those with any travel in the past year 
Agbogbloshie residents had lived in Agbogbloshie an average of 10.7 years, and 7.6 years in their current housing structure. Only $7 \%$ had moved housing structures within the past year. Overall, men had migrated a shorter distance, and had lived in Agbogbloshie and their current structure longer than women. By region, 31.9\% of men but only $19.3 \%$ of women were from Greater Accra. In terms of mobility and travel, residents had an average of 7.3 trips in the past 12 months, with women reporting more travel than men. Among those that traveled, $16 \%$ had one or more trips lasting at least a month, and $27 \%$ said that they traveled regularly. Both long-term and regular travel were more common among women than men.

Also in Table 2, we present the sexual history characteristics of the population. Men had a mean 6.4 lifetime sexual partners, compared to 2.9 for women. The age of sexual debut was similar for men (18.7 years of age) and women (17.9). In terms of cumulative number of partners over the past year, two-thirds had one partner, but single partnerships were more highly reported by women (77.1\%) than men (52.2\%). Men in general had a significantly higher $(p<0.001)$ number of cumulative partners $(1.69)$ than women (1.00). Fourteen percent of men had three or more partners, as did $4 \%$ of women. Our calendar-based sexual partnership module was limited to the previous three partners in the past year, resulting in truncation of data for the $7 \%$ of men and $1 \%$ of women who had four or more partners.

The number of unprotected partners in the past year was defined as those sexual partnerships in which any unprotected sexual intercourse (i.e., sex without a condom) occurred. The distribution of unprotected partner number was slightly lower than for total partners, since some partnerships were fully protected. The proportion of men in a single unprotected partnership is higher than the proportion in any partnership (58\% versus $52 \%$ ), and the reverse is true for women (72\% versus $77 \%)$. Presented another way, $75 \%$ of men and $91 \%$ of women had unprotected sex in all their partnerships, and $18 \%$ of men and $2 \%$ of women had unprotected sex in some of their partnerships (however, it is impossible to be in this latter category unless one has had at least two partners, so it partially reflects sex differences in the total degree). Alternatively, men had an average of 1.22 unprotected partners in the past year, compared to 0.93 mean unprotected partners for women. Given the mean number of total partners above, the ratio of total mean partners to unprotected mean partners was 1.39 for men and 1.09 for women. This provides evidence of greater condom use by men than women with partner number held constant.

Whereas these partnership distributions represent the total or cumulative partnerships over the past year, we also asked about the point distribution at each month to assess the longitudinal changes within the year. This is important to know for the $42 \%$ of men and $18 \%$ of women who had varying partnership degree over the year. Figure 3 plots the time-series of degree distributions for that subset with a changing 
degree, with individual panels for those with a maximum degree of one (46\%), two $(40 \%)$, or three $(14 \%)$. Men are represented in blue and women in red, and the line thickness is proportional to the subject's age. Few women reported having two or three partnerships at any time in the last year (see the red lines in the second and third panel in figure 3), and the degree of most men and women who report a maximum of two partners varies between one and two partnerships, while rarely dropping to zero (second panel in figure 3).

Figure 3: Change in partnership degree distributions across past 12 months, among subjects with a changing degree

\section{Changed degree $(\max$ of 1$)$}

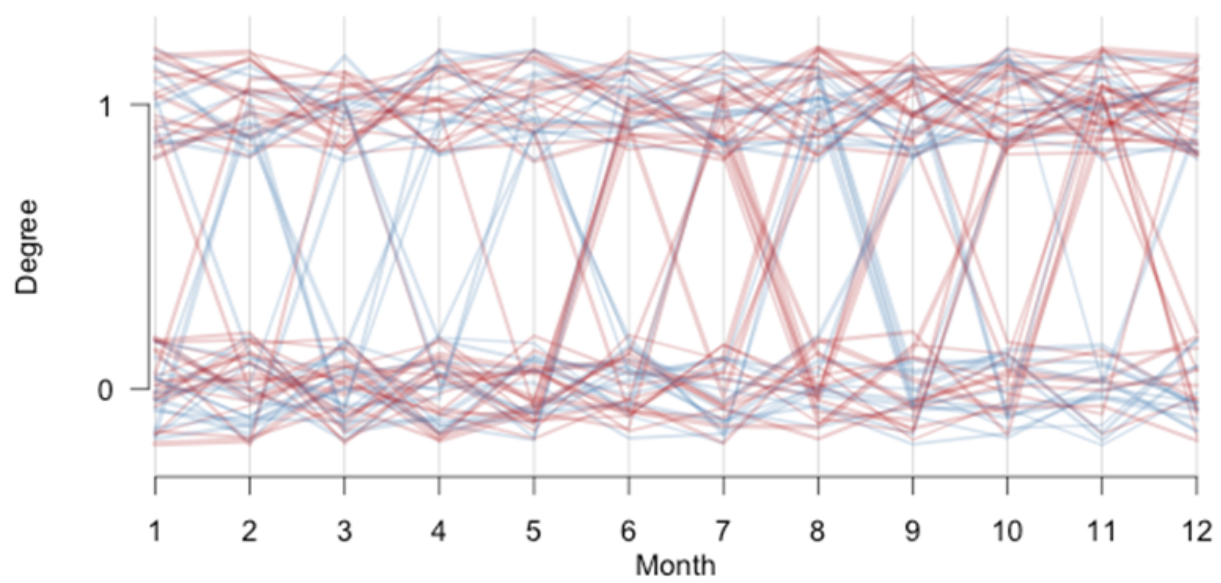


Figure 3: (Continued)

Changed degree (max of 2)

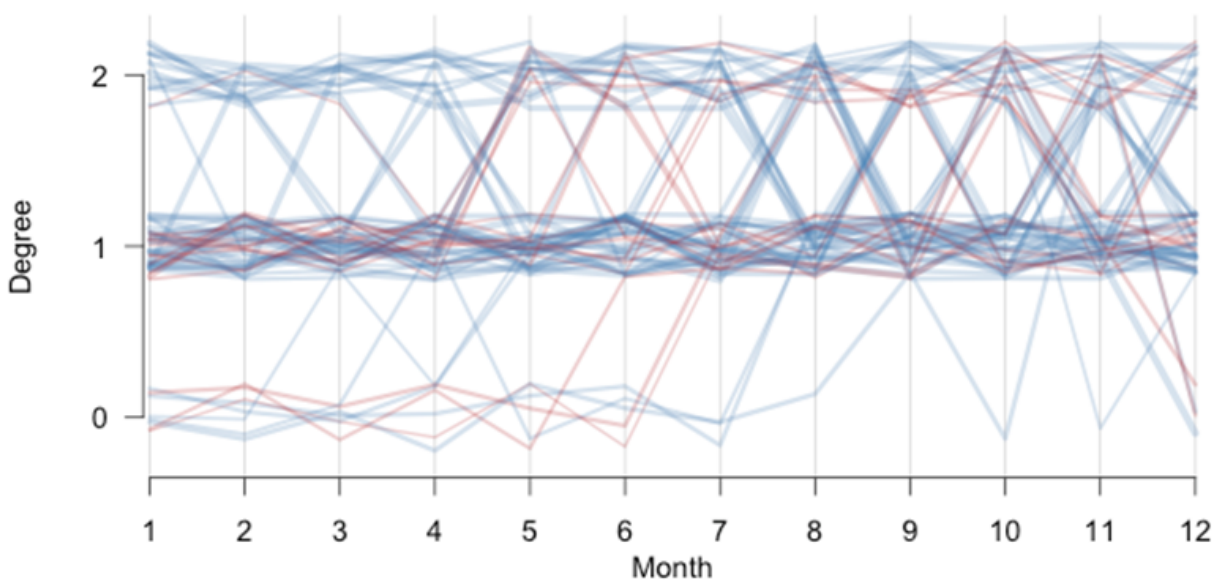

Changed degree (max of 3)

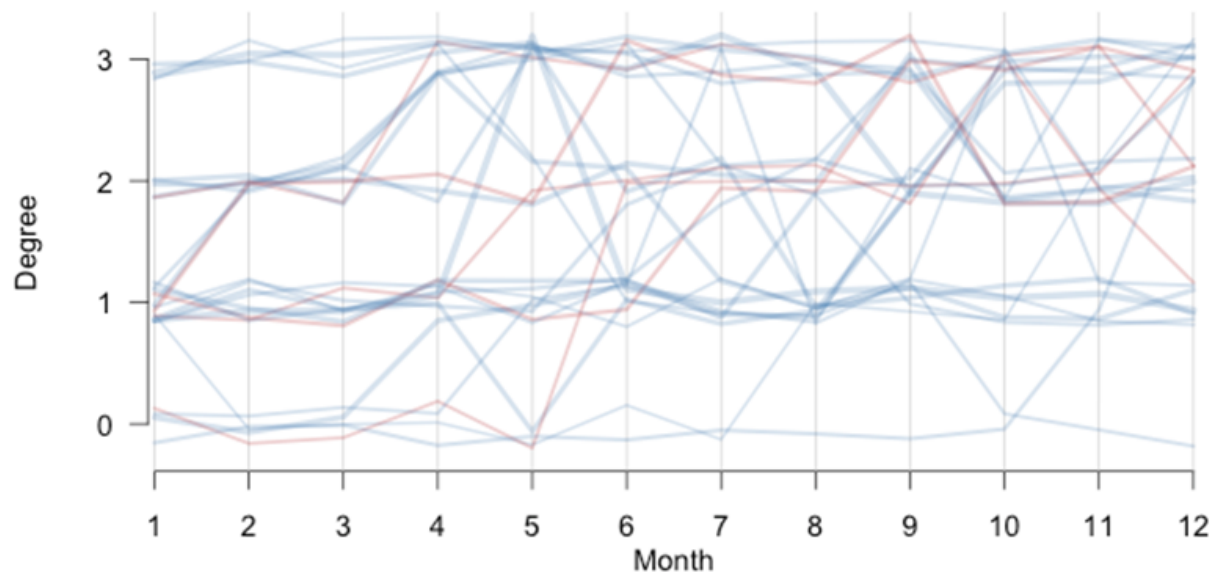

\section{Discussion}

The MHG study was the first demographic and epidemiologic study of Agbogbloshie to estimate prevalent HIV-1/2 infection. It is one of the first within Africa to characterize 
the complex interrelationships of sexual network structure, migration, and mobility, and HIV prevalence. Despite the challenging research environment, we achieved a scientifically and statistically rigorous population-based sample of the target population. The prior work of the Edulink study and the experience and skill of the field and support staff were instrumental to our success. The current and forthcoming analyses from this study will help inform HIV prevention efforts in the region.

Our key epidemiologic result was the prevalence of HIV-1/2 in the target population. We found a prevalence of HIV-1 (5.5\%) that is over twice the national prevalence of $2.1 \%$ and nearly twice the prevalence within the Greater Accra region (3.2\%). Consistent with national estimates (Ghana AIDS Commission 2012) and similar to epidemiologic profiling throughout sub-Saharan Africa (UNAIDS 2012), our observed prevalence among women (7.2\%) was over twice that of men (2.8\%). Interestingly, we observed no HIV-2 infection in our sample. Although we may have observed some HIV-2 cases if our sampling fraction of the target population were larger, our results were consistent with prior Ghana DHS analyses showing that the vast majority (95\%) of HIV infections in Ghana were with the HIV-1 type. It is plausible that our Agbogbloshie target population may be even less likely to contract HIV-2 infection given the high mobility of the population.

In future analyses we will investigate the demographic and behavioral characteristics associated with HIV infection. However, the principal limitation to that is that our measure of HIV infection is prevalence and not incident infection, so the standard limitations of inferring predictors of infection from predictors of disease maintenance (i.e., length-biased sampling) are present.

Patterns of sexual behavior data in Agbogbloshie tend to reflect higher risk compared to the 2008 Ghana DHS data. For instance, age of sexual debut for men and women, aged 20-49 in the GDHS, was 20.1 and 18.4 years respectively (Ghana Statistical Service (GSS) 2009), whereas the age of sexual debut for men and women in Agbogbloshie was slightly lower at 18.7 and 17.9 years. Additionally, the distribution of number of partners over the last 12 months was higher for residents in Agbogbloshie compared to the GDHS estimates. A much higher proportion of Agbogbloshie residents reported two or more partners in the last year compared to GDHS estimates: $37.2 \%$ compared to around $14 \%$ of men, and $8.9 \%$ compared to just over $1 \%$ of women. Higher levels of sexual risk behavior in our survey could be because residents of Agbogbloshie actually engage in higher risk, or because our event-history calendar is better able to accurately collect sensitive data compared to the DHS. Our event-history calendar data are also able to represent both the cumulative number of partnerships and number at the day of the interview. Both measures have been used to define concurrency and other sexual network features (UNAIDS Reference Group 2010), but fail to show the complexities of how the partnership degree varies over time. 
The majority of Agbogbloshie residents were not born in the Greater Accra region, suggesting that Agbogbloshie residents are more likely to have ever moved compared to national estimates in Ghana. According to 2008 Ghana DHS data, about two-thirds of women had ever moved in their lifetime, compared to slightly more than half of men. Consistent with global migration trends (International Organization for Migration 2013), we found that women in our study not only were quite mobile, but short-term mobility among women was higher compared to that of men. Women in our study reported on average a higher number of overnight trips a year compared to men, and both men and women in our study reported a higher number of overnight trips compared to the Ghana DHS (Ghana Statistical Service (GSS) 2009). Women were also slightly more likely than men to have traveled away from home for more than a month in the last year.

Residents in Agbogbloshie come from all ten regions in Ghana, but the proportions of men and women from each region differ significantly. Almost one third of male respondents were born in the Greater Accra region, compared to less than $20 \%$ of women. For comparison, $16 \%$ of all Ghanaians currently live in the Greater Accra region (Ghana Statistical Service 2012). A higher proportion of female respondents were born in the Central and Northern Regions compared to men. The Upper East region seems to be disproportionately represented in Agbogbloshie: nationally, 4.2\% of Ghanaians live in the Upper East Region (Ghana Statistical Service 2012), but nearly $10 \%$ of Agbogbloshie respondents were born there.

Many studies sampling potentially hidden populations in dense and complex urban environments use convenience sampling for recruitment. The principal drawback is that no inference to the underlying target population is possible. We benefitted from the ongoing population enumeration work of Agbogbloshie through the Urban Health and Poverty in Accra study to obtain a population-based sample (Dodoo 2012). However, this was not without its challenges. Agbogbloshie is a dynamic environment, and many of the people and places had changed considerably since the 2010 census from which we drew our sample. Flooding and fires had displaced a considerable portion of the population, although many had returned at the time of our survey. We based our household selection probabilities on the enumerated household size in the census, but this proved inaccurate for two reasons: first, there was significant residential migration resulting in inaccuracies in 2012; second, our target population of sexually active adults was not the same as the census population of all household members. Although we attempt to redress this issue through a reweighting of the sample, there is still potential for residual bias in our results related to these changes from the 2010 census.

Our survey design has allowed us to capture dynamic attributes of recent mobility and sexual partnerships, while minimizing the common limitations of selection bias and 'lost to follow-up' seen in prospective cohort studies (Rothman, Greenland, and Lash 
2008). Cross-sectional studies with retrospective, time-varying surveys can measure the timing, frequency, and duration of demographic outcomes and their changes over time. The main limitation with any retrospective measurement is the potential for recall errors and other response bias (Koepsell and Weiss 2003). However, event-history techniques like those we employed can help minimize these biases by anchoring events in time and allowing interviewers to check and reconcile inconsistencies in reports (Luke et al. 2011). A persistent limitation for epidemiologic research, however, is that serum samples are only available from the present, which limits the ability to differentiate prevalent from incident disease.

Data on sexual partnerships was obtained from summary questions for the lifetime and recent sexual history, as well as detailed questions on up to the past three partnerships in the past year. In this method, called egocentric sampling of sexual partnerships, subjects describe the features of the partnership (start and end dates) and attributes of the partners, as well as epidemiologically relevant behaviors like frequency of sex acts and condom use (Morris 2004). For network epidemiology applications that investigate how the structural elements of the network impact disease transmission, egocentric sampling allows for an inference to the full population of partnerships that are contained within the target population without the daunting task of tracking down all partners within a network (called sociocentric sampling) (Morris and Kretzschmar 1997).

\subsection{Future work}

Our forthcoming analyses from this project will focus first on standard statistical estimation of population-level characteristics and associations, and second on networkbased mathematical models for HIV transmission with model parameters derived from the primary analyses. Other analyses from this work will capitalize on the rich longitudinal data structure of the sexual partnership module to understand the timevarying patterns in behavior. Our linked partnership data were not highlighted in this paper, but will enable some important analysis of sexual partnership data reported on one partnership by its two members.

\subsection{Limitations}

Some of the limitations of the MHG study concern the standard measurement issues in social epidemiology research, such as the potential for poor recall of past events or intentional misreporting of sensitive outcomes. So long as such biases are non- 
differential with respect to comparison groups this will tend to conservatively bias any comparative statistics, but would have unforeseen implications for descriptive parameter estimation to be used in mathematical modeling. Our linked partner data will also be used to validate the reports on sexual behavior that may be subject to these biases. Second, the MHG target population represents a small geography, and many study subjects had partnerships outside of it. This represents a boundary specification problem that may have important implications for the network-based models parameterized with estimates from this population. Methods for dealing with these issues are currently being considered, but for the time being this requires an assumption of homogeneity between the measured and unmeasured components of the population.

\subsection{Conclusions}

Population-based surveys of migration and sexual risk behavior using relationship history calendars in low-resource settings are possible and produce high quality data. Residents in Agbogbloshie are disproportionately affected by HIV, and have high levels of short-term mobility. The majority of residents moved from a different region in Ghana, but contrary to popular belief, stay in Agbogbloshie for many years. HIV prevention interventions targeted at highly mobile populations in low-resource settings may have far-reaching and long-term implications.

\section{Acknowledgements}

We would like to thank the study participants and the following for their invaluable assistance of this project: Kamil Fuseini, Fidelia Dake and the staff at the Regional Institute for Population Research at the University of Ghana, the Ghana AIDS Commission, our interview team: Vincent Kantah, Patrick Nyarko, Charlotte Ofori, Cecilia Segbedji, Maame Yaa Konamah Siaw, Bilaal Tackie, Habakkuk Tarezina, Solomon Tetteh, and the laboratory staff at NMIMR: Prince Parbie and Joyce AppiahKubi. This work was supported in part by the NICHD (R00 HD057533) and the UW Center for AIDS Research SPRC (P30 AI027757). Computing support was provided by a NICHD research infrastructure grant (5R24HD042828), to the UW Center for Studies in Demography \& Ecology. 


\section{References}

AduSarkodie, Y., Tetteh, C., and AppiahDenkyira, E. (1996). Infrequent occurrence of HIV-2 in blood donors and pregnant women in a rural community in Ghana. Sexually Transmitted Diseases 23(6): 526-527. doi:10.1097/00007435199611000-00017.

Agyei-Mensah, S. (2001). Twelve years of HIV/AIDS in Ghana: Puzzles of Interpretation. Canadian Journal of African Studies 35(3): 441-472. doi:10.2307/486296.

Agyei-Mensah, S. and Oteng-Ababio, M. (2012). Perceptions of health and environmental impacts of e-waste management in Ghana. International Journal of Environmental Health Research 22(6): 500-517. doi:10.1080/09603123.2012.667795.

Akwara, P.A., Fosu, G.B., Govindasamy, P., Alayón, S., and Hyslop, A. (2005). An InDepth Analysis of HIV Prevalence in Ghana: Further Analysis of Demographic and Health Surveys Data. Calverton, Maryland, USA: ORC Macro.

Anarfi, J.K. (1993). Sexuality, migration and AIDS in Ghana-A socio-behavioral study. Health Transit Rev 3(^ sSup).

Anglewicz, P. (2012). Migration, Marital Change, and HIV Infection in Malawi. Demography 49(1): 239-265. doi:10.1007/s13524-011-0072-x.

Arguello, J.E.M., Grant, R., Oteng-Ababio, M., and Ayele, B.M. (2013). Downgrading An overlooked reality in African cities: Reflections from an indigenous neighborhood of Accra, Ghana. Applied Geography 36: 23-30. doi:10.1016/j.apgeog.2012.04.012.

Awumbila, M. and Ardayfio-Schandorf, E. (2008). Gendered poverty, migration and livelihood strategies of female porters in Accra, Ghana. Norsk Geografisk Tidsskrift-Norwegian Journal of Geography 62(3): 171-179. doi:10.1080/00291950802335772.

Brandful, J.A.M., Ampofo, W.K., Apeagyei. F.A., AsareBediako, K., and OseiKwasi, M. (1997). Predominance of HIV-1 among patients with AIDS and AIDSrelated complex in Ghana. East African Medical Journal 74(1): 17-20. 
Brandful, J.A.M., Ampofo, W.K., Janssens, W., Adu-Sarkodie, Y., Apeagyei, F., Anyomi, F., Aidoo, S., Yamamoto, N., Ishikawa, K., Sata, T., and Kurata, T. (1998). Genetic and phylogenetic analysis of HIV type 1 strains from southern Ghana. Aids Research and Human Retroviruses 14(9): 815-819. doi:10.1089/aid.1998.14.815.

Caldwell, J.C. (1969). African rural-urban migration: The movement to Ghana's towns. Columbia University Press.

Camlin, C.S., Kwena, Z.A., Dworkin, S.L., Cohen, C.R., and Bukusi, E.A. (2014). "She mixes her business": HIV transmission and acquisition risks among female migrants in western Kenya. Social Science \& Medicine 102: 146-156. doi:10.1016/j.socscimed.2013.11.004.

Cassels, S., Jenness, S.M., and Khanna, A. (2013). Conceptual Framework and Research Methods for Migration and HIV Transmission Dynamics. AIDS \& Behavior 2013(21 Nov). doi:10.1007/s10461-013-0665-z.

Cassels, S., Manhart, L., Jenness, S.M., and Morris, M. (2013). Short-term Mobility and Increased Partnership Concurrency among Men in Zimbabwe. Plos One 8(6): e66342. doi:10.1371/journal.pone.0066342.

Cote, A.M., Sobela, F., Dzokoto, A., Nzambi, K., Asamoah-Adu, C., Labbe, A.C., Masse, B., Mensah, J., Frost, E., and Pepin, J. (2004). Transactional sex is the driving force in the dynamics of HIV in Accra, Ghana. Aids 18(6): 917-925. doi:10.1097/00002030-200404090-00009.

de Silva, T., Leligdowicz, A., and Rowland-Jones, S. (2012). Effect of HIV-2 infection on HIV-1 disease progression. $N$ Engl J Med 367(20): 1961-1962; author reply 1962-1963.

de Silva, T.I., van Tienen, C., Onyango, C., Jabang, A., Vincent, T., Loeff, M.F., Coutinho, R.A., Jaye, A., Rowland-Jones, S., Whittle, H., Cotton, M., and Hue, S. (2013). Population dynamics of HIV-2 in rural West Africa: comparison with HIV-1 and ongoing transmission at the heart of the epidemic. Aids 27(1): 125-134. doi:10.1097/QAD.0b013e32835ab12c.

Deane, K.D., Parkhurst, J.O., and Johnston, D. (2010). Linking migration, mobility and HIV. Tropical Medicine \& International Health 15(12): 1458-1463. doi:10.1111/j.1365-3156.2010.02647.x.

Decosas, J. (1995). Epidemic in Ghana: "a very distinct profile". AIDS Analysis Africa 5(3): 12. 
Decosas, J., Kane, F., Anarfi, J.K., Sodji, K.D.R., and Wagner, H.U. (1995). Migration and AIDS. Lancet 346(8978): 826-828. doi:10.1016/S0140-6736(95)91631-8.

Djomand, G., Greenberg, A.E., Sassan-Morokro, M., Tossou, O., Diallo, M.O., Ekpini, E., Ghys, P., Soro, B., Brattegaard, K., Yapi, A., and et al. (1995). The epidemic of HIV/AIDS in Abidjan, Cote d'Ivoire: a review of data collected by Projet RETRO-CI from 1987 to 1993. J Acquir Immune Defic Syndr Hum Retrovirol 10(3): 358-365.

Dodoo, N.D. (2012). Decision making in Ghanaian Homes: The Role of Women. Regional Institute for Population Studies, University of Ghana.

Duda, R.B., Darko, R., Adanu, R.M.K., Seffah, J., Anarfi, J.K., Guatam, S., and Hill, A.G. (2005). HIV prevalence and risk factors in women of Accra, Ghana: Results from the women's health study of Accra. The American Society of Tropical Medicine and Hygiene 73(1): 63-66.

Ghana, AIDS Commission (2012). Ghana Country AIDS Progress Report: January 2010 - December 2011.

Ghana, Statistical Service (2012). 2010 Population and Housing Census: Summary Report of Final Results.

Ghana, Statistical Service (GSS) (2009). Ghana Demographic and Health Survey 2008. Accra, Ghana: GSS, GHS, and ICF Macro.

Goodreau, S.M., Carnegie, N.B., Vittinghoff, E., Lama, J.R., Sanchez, J., Grinsztejn, B., Koblin, B.A., Mayer, K.H., and Buchbinder, S.P. (2012). What Drives the US and Peruvian HIV Epidemics in Men Who Have Sex with Men (MSM)? PLoS One 7(11): e50522. doi:10.1371/journal.pone.0050522.

Hugo, P. (2010). A Global Graveyard for Dead Computers in Ghana. New York Times.

International Organization, for Migration (2013). World Migration Report 2013.

Ishikawa, K., Tsujimoto, H., Nakai, M., Mingle, J.A.A., Oseikwasi, M., Aggrey, S.E., Nettey, V.B.A., Afoakwa, S.N., Fukasawa, M., Kodama, T., Kawamura, M., and Hayami, M. (1988). Isolation and Characterization of Hiv-2 from an Aids Patient in Ghana. Aids 2(5): 383-388. doi:10.1097/00002030-198810000-00009.

Joint UNEP/OCHA, Environment Unit (2011). Rapid Disaster Waste Management Assessment 26 October Flash Flooding, Central Accra - Ghana.

Kahle, D. and Wickham, H. (2013). ggmap: Spatial Visualization with ggplot2. The $R$ Journal 5: 144-161. 
Koepsell, T.D. and Weiss, N.S. (2003). Epidemiologic Methods: Studying the Occurence of Illness. New York, NY: Oxford University Press.

Kumi-Kyereme, A., Tuoyire, D.A., and Darteh, E.K. (2013). Determinants of Unprotected Casual Heterosexual Sex in Ghana. Journal of Biosocial Science: 1-19.

Luke, N., Clark, S., and Zulu, E. (2011). The Relationship History Calendar: Improving the Scope and Quality of Data on Youth Sexual Behavior. Demography 48: 1151-1176. doi:10.1007/s13524-011-0051-2.

Lumley, T. (2004). Analysis of complex survey samples. Journal of Statistical Software 9(8): 1-19.

Masuda, T., Tsujimoto, H., Ishikawa, K., Ohta, Y., Hayami, M., Ocheng, E.M., Johnson, B.K., Tukei, P.M., Delaporte, E., Cooper, R.W., Frost, E., Mingle, J.A.A., and Oseikwasi, M. (1988). Reactivities of Antibodies to Hiv and Siv in Human-Sera in Kenya, Gabon, and Ghana. Lancet 1(8580): 297. doi:10.1016/S0140-6736(88)90375-3.

Morris, M. (2004). Network Epidemiology: A handbook for survey design and data collection. Oxford: Oxford University Press. doi:10.1093/0199269017.001.0001.

Morris, M., Goodreau, S.M., and Moody, J. (2007). Sexual Networks, Concurrency, and STD/HIV. In: Holmes, K.K.: Sexually Transmitted Diseases. New York: McGraw-Hill: 109-126.

Morris, M. and Kretzschmar, M. (1997). Concurrent partnerships and the spread of HIV. AIDS 11(5): 641-683. doi:10.1097/00002030-199705000-00012.

Nyame, F.K., Grant, J.A., and Yakovleva, N. (2009). Perspectives on migration patterns in Ghana's mining industry. Resources Policy 34(1-2): 6-11. doi:10.1016/j.resourpol.2008.05.005.

Oberhauser, A.M. and Yeboah, M.A. (2011). Heavy burdens: Gendered livelihood strategies of porters in Accra, Ghana. Singapore Journal of Tropical Geography 32(1): 22-37. doi:10.1111/j.1467-9493.2011.00417.x.

Oppong, J.R. (1998). A vulnerability interpretation of the geography of HIV AIDS in Ghana, 1986-1995. Professional Geographer 50(4): 437-448. doi:10.1111/0033-0124.00131.

Oteng-Ababio, M. (2010). E-waste: an emerging challenge to solid waste management in Ghana. International Development Planning Review 32(2): 191-206. doi:10.3828/idpr.2010.02. 
Oteng-Ababio, M. (2012). When Necessity Begets Ingenuity: E-Waste Scavenging as a Livelihood Strategy in Accra, Ghana. African Studies Quarterly 13(1\&2).

Owusu, M. (1995). Nigeria. In: Ghana: a country study. Library of Congress.

Potts, D. (2009). The slowing of sub-Saharan Africa's urbanization: evidence and implications for urban livelihoods. Environment and Urbanization 21(1): 253-259. doi:10.1177/0956247809103026.

Potts, D. (2012). What do we know about urbanisation in sub-Saharan Africa and does it matter? International Development Planning Review 34(1): V-Xxi. doi:10.3828/idpr.2012.1.

Rothman, K.J., Greenland, S., and Lash, T.L. (2008). Modern Epidemiology. Philadelphia: Wolters Kluwer Health.

Stock, R. (2013). Africa South of the Sahara: A Geographical Interpretation. New York: The Guilford Press.

Tatem, A.J., Hemelaar, J., Gray, R.R., and Salemi, M. (2012). Spatial accessibility and the spread of HIV-1 subtypes and recombinants. Aids 26(18): 2351-2360. doi:10.1097/QAD.0b013e328359a904.

UNAIDS (2010). Global Report: UNAIDS Report on the Global AIDS EPidemic 2010. Geneva: UNAIDS.

UNAIDS (2012). Global report: UNAIDS report on the global AIDS epidemic 2012.

UNAIDS, Reference Group (2010). HIV: consensus indicators are needed for concurrency. Lancet 375(9715): 621-622. doi:10.1016/S0140-6736(09)62040-7.

Verutes, G.M., Fiocco, M.B., Weeks, J.R., and Coulter, L.L. (2012). Health, poverty, and place in Accra, Ghana: mapping neighborhoods. Journal of Maps 8(4): 369-373. doi:10.1080/17445647.2012.744365.

Voeten, H.A.C.M., Vissers, D.C.J., Gregson, S., Zaba, B., White, R.G., de Vlas, S.J., and Habbema, J.D.F. (2010). Strong Association Between In-Migration and HIV Prevalence in Urban Sub-Saharan Africa. Sexually Transmitted Diseases 37(4): 240-243.

Weeks, J.R., Getis, A., Hill, A.G., Agyei-Mensah, S., and Rain, D. (2010). Neighborhoods and Fertility in Accra, Ghana: An AMOEBA-Based Approach. Annals of the Association of American Geographers 100(3): 558-578. doi:10.1080/00045601003791391. 
Weeks, J.R., Getis, A., Stow, D.A., Hill, A.G., Rain, D., Engstrom, R., Stoler, J., Lippitt, C., Jankowska, M., Lopez-Carr, A.C., Coulter, L., and Ofiesh, C. (2012). Connecting the Dots Between Health, Poverty, and Place in Accra, Ghana. Annals of the Association of American Geographers 102(5): 932-941. doi:10.1080/00045608.2012.671132.

Weeks, J.R., Hill, A., Stow, D., Getis, A., and Fugate, D. (2007). Can we spot a neighborhood from the air? Defining neighborhood structure in Accra, Ghana. GeoJournal 69(1-2): 9-22. doi:10.1007/s10708-007-9098-4.

White, M.J., Muhidin, S., Andrzejewski, C., Tagoe, E., Knight, R., and Reed, H. (2008). Urbanization and Fertility: An event-history analysis of coastal Ghana. Demography 45(4): 803-816. doi:10.1353/dem.0.0035. 
Cassels et al.: Migration, sexual networks, and HIV in Agbogbloshie, Ghana 